\title{
Adenosine-Sensitive Ventricular Tachycardia by ECG Finding
}

National Cancer Institute

\section{Source}

National Cancer Institute. Adenosine-Sensitive Ventricular Tachycardia by ECG Finding. NCI Thesaurus. Code C71057.

An electrocardiographic finding of tachycardia that is usually terminated by the administration of adenosine. Usually, it is a result of cAMP-mediated triggered activity and typically originates from the right ventricular outflow tract. This type of tachycardia occurs in patients with apparently normal hearts. 